\title{
CINQUANTE ANNÉES DE RECHERCHES EN COMMUNICATION
}

\author{
Marc Lits ${ }^{1}$
}

Combien de livres de vulgarisation et de cours de communication ne s'ouvrent-ils pas sur cette formule passe-partout : "De tout temps, en tout lieu, l'homme a toujours communiqué..." ? Si l'on prend par ailleurs en compte l'adage rendu célèbre par les théoriciens américains de l'école de Palo Alto, "On ne peut pas ne pas communiquer", il semble peu pertinent de situer les origines de la communication après la seconde guerre mondiale. Pourtant, si l'homme est par essence un animal communicant, il n'avait jamais rassemblé, jusqu'il y a peu, ses différentes théories sur les modèles d'échanges intersubjectifs, les systèmes de signes et les supports utilisés pour ce faire en un projet unique. C'est-à-dire qu'il n'avait jamais identifié comme tel un objet formel ou matériel dûment délimité (la communication) ou dessiné les contours d'une discipline plus ou moins autonome (les sciences de l'information et de la communication). Certes, Aristote a discuté de questions linguistiques, de problèmes de représentation, d'effets de communication, et d'autres, après lui, traiteront de questions de langage, de relations

1 Président du Département de communication de l'Université catholique de Louvain, Co-directeur de l'Observatoire du Récit Médiatique (ORM).

Recherches en communication, $\mathrm{n}^{\circ} 11,(1999)$. 
entre personnes ou entre groupes, de principes communicationnels, depuis saint Augustin jusqu'à Sigmund Freud, des Encyclopédistes à Gabriel Tarde. Néanmoins, on peut considérer que c'est dans la lignée du développement des sciences du langage (linguistique, sémiologie) et des sciences de l'homme (anthropologie, psychologie, sociologie...) que vont progressivement se constituer les sciences de l'information et de la communication, identifiées comme telles. Comme domaine disciplinaire autonome ou comme interdiscipline au croisement de sciences sociales et humaines de référence, puisque ce débat est encore loin d'être tranché.

\section{Le développement de la communication}

La communication peut être prise en compte selon trois aspects très différents : le vécu de la communication ordinaire sur lequel s'interroge de plus en plus le grand public (particulièrement séduit par des gourous du relationnel comme un Jacques Salomé, par exemple); la pratique de la communication des professionnels des médias, de l'entreprise, de l'éducation (qui privilégient eux les manuels du type "Savoir bien communiquer en 20 leçons") ; la théorisation critique des chercheurs en communication. Cela explique pourquoi de si nombreuses personnes se croient autorisées à parler des phénomènes de communication, et entretiennent la confusion dans le public, mais aussi dans le milieu des chercheurs qui n'ont pas tous la même conception de la recherche, fondamentale ou appliquée, conceptuelle ou gadgétisée. D'autant que l'approche scientifique elle-même est au cœur d'un dispositif beaucoup plus vaste aux multiples ramifications et spécialités. Dès lors, définir l'objet de la communication, comme nous avons voulu le tenter dans le département de communication de l'UCL au moment de publier la revue "Recherches en communication", devient un véritable risque. Qu'il faut cependant tenter pour s'entendre sur un projet collectif et un objet commun. Nous disions alors, dans l'énoncé de notre projet éditorial et scientifique, que cette science consiste en l'étude des actes de communication socialement élaborés, des significations portées par ces actes, des relations et phénomènes cognitifs sollicités par ceux-ci, des représentations socialement partagées qui s'ensuivent et des effets de représentations sur la réalité sociale. 
La communication est ainsi, au sens propre, une interdiscipline dont les théories et méthodes proviennent de champs disciplinaires voisins, historiquement antérieurs, que celle-ci adopte en les adaptant. Mais à côté de cette hétérogénéité disciplinaire, il faut aussi tenir compte des lieux d'application extrêmement diversifiés et en permanente évolution. La presse écrite, la radio, la télévision, la communication interne et externe des entreprises comme des organisations non marchandes, la vulgarisation scientifique, l'éducation aux médias : autant de secteurs spécialisés qu'il faut observer. Sans oublier les développements récents des nouvelles technologies de l'information : journalisme multimédia, gestion de sites WEB, enjeux des formations à distance. Ces nouveaux protocoles de communication, et les métiers qu'ils induisent, doivent être maîtrisés, par les nombreux professionnels du secteur, mais ils doivent aussi être analysés, pensés dans leur intégration sociale, afin que soient perçus les nouveaux paradigmes qu'ils disposent, dans l'organisation de la société comme dans la saisie cognitive individuelle du savoir.

\section{Pour une histoire des théories...}

Le temps semble venu maintenant de rédiger l'histoire de cette discipline qui tente à la fois de s'autonomiser et de se situer par rapport à des références qu'elle ne peut ni ne veut renier. Ce n'est donc pas un hasard si les ouvrages de synthèse se multiplient depuis quelques années, à la fois pour donner des bilans de vulgarisation aux étudiants inscrits en nombre de plus en plus important dans le secteur, et pour dégager les lignes de faîte d'un champ dont les contours demandent à être quelque peu clôturés. D. Bougnoux, Ph. Breton et $\mathrm{S}$. Proulx, L. Sfez, A. Mattelart, B. Miège, D. Wolton (pour respecter un strict ordre alphabétique), bien d'autres s'y sont essayés, mais en choisissant pour la plupart une approche descriptive et cumulative, empilant les modèles, théories et méthodes sans se prononcer trop ouvertement sur leur validité propre et leur apport au champ communicationnel. Il serait pourtant opportun de trouver l'équivalent d'un François Dosse qui utiliserait la méthode historique pour écrire, à l'instar de son histoire du structuralisme, une histoire des sciences de la communication. Évidemment, il serait prématuré de déjà 
entonner le chant du cygne, et plus prudent de baliser en l'état actuel le champ du signe communicationnel.

Au-delà de l'anecdote, le relevé des origines disciplinaires des principaux théoriciens de la communication dans l'espace francophone serait déjà significatif pour établir la carte du territoire, les filiations disciplinaires et l'entrée progressive de certains modèles dans le champ. En effet, la communication, ce carrefour conceptuel, ne peut se comprendre qu'en situation par rapport à sa constitution diachronique et à sa position particulière dans le secteur des sciences humaines. La nécessité d'étudier systématiquement les phénomènes liés à la communication s'est ainsi imposée au moment où les moyens de communication -en particulier les mass media- commencèrent à jouer un rôle déterminant dans l'organisation des sociétés modernes. Pour la sociologie, l'intérêt s'est alors porté sur les communications de masse considérées comme l'instrument privilégié de l'idéologie. La sémiologie structuraliste, quant à elle, a envisagé ces communications comme mues par un code de règles communes. De là naitra l'idée de la communication comme transmission d'information. Ces conceptions laissant dans l'ombre les sujets et leurs interactions, différentes théories pragmatiques tenteront de considérer la communication comme une série d'actes capables de structurer les rapports sociaux. L'approche anthropologique réfléchit, quant à elle, aux rapports entre systèmes de communication et formes d'organisation sociale, tandis que d'autres chercheurs, frappés par l'importance du rôle joué par les médias, considèrent que ceux-ci sont devenus le principal lieu de référence tant de l'imaginaire collectif que des consciences individuelles, ce qui justifie l'intérêt d'aborder la problématique de la communication par le biais des récits que tout groupe social véhicule et à travers lesquels il se construit une "identité narrative". Quant aux philosophes, ils seraient parfois tentés de reporter vers les études en communication leur liaison ancienne et conflictuelle avec la théologie. Et il faudrait encore mentionner l'éthique, le droit, voire de nouvelles tendances comme les "cultural studies", les sciences cognitives ou la médiologie. Sans oublier la logique, la rhétorique, l'histoire (des idées, des sciences et des techniques, des mentalités), les sciences politiques, l'ingénérie en télécommunication... 
A chaque étape, des spécialistes venus de disciplines de référence vont progressivement être intégrés à un secteur encore trop jeune pour s'être construit une armature disciplinaire suffisamment structurée, autorisant l'auto-reproduction ou une évolution endogène. Ce dont témoignent, par exemple, les débats parfois vifs entre chercheurs qui agitent des lieux fédérateurs comme la Société française des sciences de l'information et de la communication. De ces relations exogamiques, nous reparlerons plus avant, en en reconnaissant la richesse (la consanguinité ne menace pas encore les SIC !), mais aussi les dangers.

\section{...et un questionnement méthodologique}

La confrontation de modèles théoriques hétérogènes suppose en effet une mise en perspective de ceux-ci, pour éviter le rétrécissement de ces théories à des boîtes à outils, qui apparaîtraient comme dénuées de tout positionnement idéologique. Cet aplatissement théorique empêche parfois toute mise en perspective, toute contextualisation des modèles, qui ne peuvent être situés qu'au départ d'un surplomb idéologiquement marqué, et reconnu comme tel. L'hypothèse défendue par une équipe de recherche telle que l'Observatoire du récit médiatique (ORM) de l'UCL, pour qui la refiguration narrative passe essentiellement par les médias de masse, s'appuie ainsi sur des présupposés philosophiques développés par Paul Ricœur, mais aussi par des penseurs de la postmodernité tel Gianni Vattimo qui considère que les médias «jouent un rôle déterminant dans la naissance d'une société postmoderne» 1 et inscrivent «la société de communication généralisée» dans un système de «fabulation du monde» ${ }^{2}$. Ceci explique, pour le philosophe italien, le retour de la narrativité et de l'approche herméneutique dans les sciences humaines. De tels présupposés méritent bien sûr la discussion, mais ils doivent en tout cas être posés explicitement en préalable à tout projet d'interprétation des phénomènes sociaux de communication. Ainsi, comment les théories communicationnelles se situent-elles par rapport aux concepts encore confus de modernité, de postmodernité ou de surmodernité ? L'explosion de la communication, pour reprendre le

1 G. Vatrimo, La société transparente, Paris, Desclée De Brouwer, 1990, p. 13.

2 Ibid., p. 39. 
titre de l'essai de $\mathrm{Ph}$. Breton et $\mathrm{S}$. Proulx, signifie-t-elle "la naissance d'une nouvelle idéologie", pour reprendre cette fois leur sous-titre, ou confirme-t-elle l'émergence d'un nouveau domaine structuré du savoir savant?

S'y ajoutent les difficultés méthodologiques inhérentes aux bricolages, certes vantés par Lévi-Strauss et de Certeau, mais toujours délicats dans leur usage. Pour des analystes des médias, par exemple, la confrontation d'analyses textuelles, de méthodes ethnologiques, de données socio-économiques ne va pas sans problèmes délicats. Langages et concepts opérateurs différents (le même terme renvoyant parfois à des axiomatiques autrement découpées), objet d'étude protéiforme, options méthodologiques variant entre des entrées macro- ou microscopiques, entre des hypothèses de travail déductives ou empiriques... Autant de défis à résoudre pour des chercheurs qui veulent travailler de concert sur un même objet, en dépassant une simple juxtaposition de leurs coupes méthodologiques. Edgar Morin a bien sûr montré combien «la culture scientifique et technique, de par son caractère disciplinaire spécialisé, disjoint et compartimente les savoirs, rendant de plus en plus difficile leur mise en contexte» ${ }^{1}$. Il n'en est dès lors que plus urgent de chercher à reconstruire des saisies globalisantes des objets sociaux complexes. Ce qui est aussi le projet d'E. Morin :

La connaissance doit certes utiliser l'abstraction, mais en cherchant à se construire par référence au contexte. La compréhension de données particulières nécessite l'activation de l'intelligence générale, et la mobilisation des connaissances d'ensemble. Marcel Mauss disait: «Il faut recomposer le tout». Ajoutons : il faut mobiliser le tout ${ }^{2}$.

Il s'agit donc de construire un modèle d'appréhension et d'interprétation régi par divers horizons de référence méthodologique, sans pour autant sombrer dans une vision holistique. Il s'agit aussi d'oser réaliser des délocalisations conceptuelles et méthodologiques entre, par exemple, les théories d'analyse littéraire et l'étude de corpus de presse écrite ou de séquences filmiques. On acceptera donc

\footnotetext{
1 E. MORIN, "Le besoin d'une pensée complexe", Magazine littéraire, hors série 1996, p. 120.

2 lbid.
} 
la proposition d'un Gérard Genette, quand il veut élargir au "récit factuel" les méthodes de la "narratologie fictionnelle":

Quels que soient, au stade où nous en sommes, les mérites et les défauts de la narratologie fictionnelle, il est douteux qu'elle nous épargne une étude spécifique du récit factuel. Il est certain en tout cas qu'elle ne peut indéfiniment se dispenser d'une interrogation sur l'applicabilité de ses résultats, voire de ses méthodes, à un domaine qu'elle n'a jamais vraiment exploré avant de l'annexer silencieusement, sans examen ni justification. (...)

Il y faudrait une vaste enquête à travers des pratiques comme l'Histoire, la biographie, le journal intime, le récit de presse, le rapport de police, la narratio judiciaire, le potin quotidien, et autres formes de ce que Mallarmé appelait l'"universel reportage"1.

C'est ce que tente aussi André Gaudreault en réutilisant le modèle narratologique pour l'étude du cinéma ${ }^{2}$. Puisque ces chercheurs nous confortent dans notre logique de délocalisation, nous pouvons aussi ajouter que celle-ci présente quelques intérêts, dont le moindre n'est pas de dépasser le stade descriptif et analytique pour insérer ces méthodologies dans une pratique sociale. L'impureté ${ }^{3}$ de cette démarche sémiologique amène tout naturellement des chercheurs à transformer celle-ci en une nouvelle interdiscipline, que Teun Van Dijk a appelée socio-sémiotique. Mauro Wolf a ainsi montré les limites, pour les recherches en communication, des approches de type sémiotique stricto sensu, voire même sociologique, dans la mesure où elles se sont construites au départ de l'observation de textes littéraires, et ont trop souvent ignoré l'insertion sociale de ces textes :

Le souci de dégager une pertinence sémiotique l'emportait sur tout intérêt réel pour l'objet analysé (le message des médias). Cet objet pouvait se présenter indifféremment comme un programme de télévision, un article de journal, une publicité, un texte populaire ou littéraire, ou encore, une œuvre artistique. Les médias étaient ici des véhicules de production

1 G. GenetTe, Fiction et diction, Paris, Éd. du Seuil, coll. Poétique, 1991, pp. 66-67.

2 A. Gaudreault, Du littéraire au filmique. Système du récit, Paris, Klincksieck, 1988.

3 Au sens où l'entend G. SCARPETTA dans son essai précisément intitulé L'impureté, Paris, Grasset, coll. Figures, 1985. 
et de distribution des messages et des textes. L'analyse ne portait donc ni sur les médias ni sur leur fonction, mais sur des objets sémiotiques ${ }^{1}$.

Il propose dès lors de mieux saisir les médias dans leur «rôle spécifique d'intermédiaires symboliques collectifs», en appréhendant leur «langage non seulement en termes de structures formelles, mais également comme une donnée sociale» ${ }^{2}$. Place doit être accordée à une socio-sémiotique qui mettra, dans la lignée des travaux de Teun Van Dijk, «l'accent sur les conséquences (diverses, complexes, inattendues) du processus de communication de masse et sur l'importance fondamentale de la dynamique de la signification». Pour ce dernier, «le discours n'est pas seulement un objet verbal, mais essentiellement une forme d'interaction sociale ${ }^{3}$. Le propre des sciences de la communication réside probablement dans ce perpétuel entrecroisement imposé par la prise en compte de l'analyse des objets dans leur insertion sociale. En témoigne, significativement, l'efflorescence des déterminations disciplinaires mixtes: sémiopragmatique, sociosémiotique, psychosociologie, ethnosociologie, ethnonarratologie. Autant de signes de croisements féconds et fécondants, dont témoigne à sa manière la réflexion de Patrick Charaudeau :

L'étude du sens social à travers la mise en œuvre des signes est chose complexe (...). Du coup, les moyens à utiliser pour l'analyser ne peuvent relever d'une seule discipline. D'ailleurs, à notre époque, les sciences humaines et sociales se caractérisent par une forte spécialisation (elles deviennent de plus en plus «dures») et en même temps par une tentative de connexion entre disciplines différentes pour essayer de rendre compte de cette complexité 4 .

C'est en ce sens que l'approche narratologique, pour rester dans l'exemple de l'analyse des médias, ne devient signifiante qu'insérée entre, en amont, une connaissance plus complète des conditions de production de cet objet social que sont les médias, et, en aval, la

1 M. WOLF, "Recherche en communication et analyse textuelle", Hermès, n'11-12, 1993, p. 213.

2 lbid.

3 Ibid.

4 P. CHARAUDEAU, Le discours d'information médiatique. La construction du miroir social, Paris, Nathan/INA, coll. Médias/Recherche, 1997, p. 6. 
compréhension des interactions qui s'opèrent entre un média et son public. L'hybridation de cet objet a donc entraîné nécessairement l'hybridation des approches, mais a aussi permis l'émergence de cette interdiscipline, dont rien, jusqu'à présent, ne permet d'imaginer qu'elle pourrait à son tour être exportée vers d'autres domaines d'observation. Ainsi, à la volonté de Genette d'exporter son modèle vers d'autres objets, pour en vérifier la validité, répondrait la volonté des sciences de la communication de renvoyer les modèles importés vers leurs concepteurs originels, mais dans une version remaniée, voire améliorée. Le modèle construit dans une équipe comme l'ORM autour précisément de l'objet "récit médiatique", pourrait ainsi être réexporté vers ses champs théoriques d'origine, par exemple pour inviter les théoriciens du texte à revisiter leur définition du récit. Mais cela présupposerait que soient d'abord réglés quelques problèmes liés à ce type d'objet et aux méthodes utilisées.

Les premiers problèmes sont bien sûr d'ordre méthodologique. Ils portent, assez classiquement, sur l'adéquation entre le modèle construit et l'objet empirique saisi, et corrélativement, sur la priorité à déterminer (pour autant qu'il faille la déterminer) entre approche inductive et déductive. Autrement dit, peut-on concilier une prédétermination conceptuelle et "l'empirisme irréductible" qui guide nombre de recherches en communication. «L'accumulation de vérifications empiriques d'une hypothèse centrale» que critique Ien Ang fait-elle avancer la connaissance des objets et de leur usage social. Quelle est finalement la pertinence scientifique de l'accumulation d'observations ponctuelles ?

Est aussi latente, derrière ces questions, l'interrogation lancinante sur la validité des approches de type macroscopique, englobant dans une lecture nécessairement large et peut-être floue, un corpus considérable, et celle des approches de type microscopique qui ne pourraient que décrire de manière entomologique tel mécanisme spécifique, ou procéder selon le paradigme de l'indice que Carlo Ginzburg développe dans ses travaux de micro-histoire ${ }^{2}$.

1 Pour reprendre l'expression d'O. SChwartz longuement discutée par G. DEREzE dans son article "A propos de «l'empirisme irréductible»", Recherches en communication, $\mathrm{n}^{\circ} 3$, 1995, pp. 209-224.

2 C. GinzBurg, Mythes, emblèmes, traces: morphologie et histoire, Paris, Flammarion, coll. Nouvelle bibliothèque scientifique, 1989. 
Enfin, quelle est la validité et la pertinence de ces délocalisations conceptuelles et méthodologiques? Quel est leur intérêt si elles n'ont valeur que pour l'objet spécifique? Permettent-elles, après transformation, de réinterroger les disciplines d'origine, par mouvement de réexportation, dans la définition de leurs champs d'étude et de leurs méthodologies, dans la délimitation de leurs corpus? Autant de questions qui devront être approfondies pour permettre à la recherche en communication de s'appuyer sur des bases aussi clairement définies qu'indiscutables.

\section{0 années d'analyse}

Ce bilan critique de 50 années d'études en communication a été réalisé à l'occasion d'un colloque qui a réuni quelques spécialistes issus de disciplines différentes à Louvain-la-Neuve, le 25 octobre 1997, afin, précisément, de fêter le cinquantième anniversaire des études en journalisme et communication dans cette université. C'est en effet dès 1946 que l'Université catholique de Louvain ouvrait un programme d'étude dans ce secteur, ce qui représentait probablement une première européenne. Le colloque a donné l'occasion à des spécialistes français, québécois et belges, théoriciens de la sémiotique, de la pragmatique, de la narratologie, de l'anthropologie de dresser le panorama de cinq décennies de recherche. Proposition leur fut faite de dresser le bilan de leur secteur, au départ des disciplines qui nous semblèrent s'être imposées particulièrement durant chaque décennie. C'est ainsi que l'analyse de contenu et les schémas diffusionnels nous paraissent témoigner des recherches dominantes dans la période préstructurale des années 50 . Jules Gritti, qui fera partie des auteurs du fameux numéro 8 de la revue Communications consacré à l'analyse structurale des récits, nous a apporté sa vision synthétique de ces premiers temps de recherche. Ce fut malheureusement une de ses dernières interventions publiques, et l'un de ses derniers articles scientifiques. Nous voudrions rendre ici hommage à la mémoire de celui qui joua un rôle important dans la constitution du champ de l'analyse des médias, qui œuvra comme consultant dans plusieurs organes médiatiques, qui fut aussi professeur invité durant plusieurs années dans notre département de communication et y forma une génération de chercheurs. 
Pour les années 60 , il nous semblait évident de retenir l'avancée de la sémiologie, comme modèle dominant, et de donner la parole pour ce faire à un participant assidu des séminaires de Barthes et de Greimas, Herman Parret. Des années 70, nous retiendrons la prise en compte progressive des conditions de réception, qui sont particulièrement étudiées par les ethnologues et les anthropologues, ce dont témoignent Jean Lohisse et Serge Proulx. Ce mouvement de réintroduction du sujet, du consommateur de médias socialement inscrit, est aussi manifeste, dans les années 80 , à travers l'évolution des approches sémiologiques et linguistiques, lesquelles vont déplacer leurs analyses de l'énoncé vers l'énonciation, avec l'émergence de la pragmatique, synthétisée par Jean-Pierre Meunier et Daniel Bougnoux. Enfin, la dimension narrative, considérée comme l'un des enjeux du système postmoderne, est présentée par Philippe Marion et André Gaudreault, qui s'intéressent tous deux, durant les années 90, à l'émergence d'une narratologie médiatique et transmédiatique. Bien sûr, cette périodisation est éminemment arbitraire, la question du récit, par exemple, étant déjà présente dans les modèles structuraux des années 60, tandis que l'intérêt pour le "lecteur réel" est simultanément reconnu par les ethnologues et les linguistes de l'énonciation. Ce découpage a en partie été organisé parce qu'il reflète l'émergence de ces disciplines dans les recherches et les enseignements de notre département de communication, où différentes réformes du programme ont signalé cette évolution scientifique, où la création de groupes de recherche a témoigné de l'émergence de nouveaux paradigmes contribuant à construire et à modifier les sciences de l'information et de la communication. Mais ce parcours n'a rien d'exceptionnel ou de marginal, il nous semble même assez exemplaire de démarches parallèles apparues simultanément en France, au Québec, voire dans les pays anglophones. C'est à ce titre qu'il a valeur démonstrative de l'édification progressive, fluctuante, de notre secteur. 